\title{
LAW EQUIVALENCE OF ORNSTEIN-UHLENBECK PROCESSES DRIVEN BY A LÉVY PROCESS
}

\author{
GRZEGORZ BARTOSZ AND TOMASZ KANIA
}

\begin{abstract}
We demonstrate that two Ornstein-Uhlenbeck processes, that is, solutions to certain stochastic differential equations that are driven by a Lévy process $L$ have equivalent laws as long as the eigenvalues of the covariance operator associated to the Wiener part of $L$ are strictly positive. Moreover, we show that in the case where the underlying Lévy process is a purely jump process, which means that neither it has a Wiener part nor the drift, the absolute continuity of the law of one solution with respect to another forces equality of the solutions almost surely.
\end{abstract}

\section{IntroduCtion AND THE MAIN RESUlT}

Let $H$ be a real separable Hilbert space and let $L=\left(L_{t}\right)_{t \geqslant 0}$ be an $H$-valued Lévy process. We fix two bounded linear operators $A, \tilde{A}$ on $H$; should the space $H$ have finite dimension $d$, we think of $A$ and $\tilde{A}$ as $d \times d$ real matrices in some fixed basis. We consider the corresponding $H$-valued Ornstein-Uhlenbeck processes driven by a Lévy process, that is, solutions to the following stochastic differential equations:

$$
\left\{\begin{array}{l}
\mathrm{d} X_{t}=A X_{t} \mathrm{~d} t+\mathrm{d} L_{t}, \\
\mathrm{~d} \tilde{X}_{t}=\tilde{A} \tilde{X}_{t} \mathrm{~d} t+\mathrm{d} L_{t}
\end{array}\right.
$$

with the initial conditions $X_{0}=\tilde{X}_{0}=0$. (The reader will find all the required definitions as well as proofs of the results to be presented in the subsequent sections.)

It is customary to view sample paths of such processes (truncated to some initial interval $[0, T]$ for $T>0)$ as elements of the Hilbert space $H_{T}:=L_{2}([0, T], H)$. On the other hand, we may be more restrictive and regard these processes as random variables assuming values in $\mathcal{D}_{H, T}$, the space of $H$-valued càdlàg functions on $[0, T]$ furnished with the Skorohod topology. This topology is induced by the so-called Skorohod metric; the Borel $\sigma$-algebra

Date: May 14, 2019.

2010 Mathematics Subject Classification. 60H10, 60G15 (primary), and 93E11, 60G30 (secondary).

Key words and phrases. SDE, Lévy process, Ornstein-Uhlenbeck process, Lévy-Itô decomposition, Wiener process, Girsanov's theorem.

The second-named author acknowledges with thanks funding received from GAČR project 19-07129Y; RVO 67985840 (Czech Republic). 
of $\mathcal{D}_{H, T}$ coincides then with the cylindrical $\sigma$-algebra, that is, the smallest $\sigma$-algebra making the point evaluations

$$
p_{t}(f)=f(t) \quad\left(t \in[0, T], f \in \mathcal{D}_{H, T}\right)
$$

measurable-this is an important feature unavailable in the Hilbert space $H_{T}$. A natural question then arises.

In which circumstances such two processes have equivalent laws?

This line of research concerning the study of equivalence of laws was initiated by Kozlov ([11]) in the setting where $A, \tilde{A}$ are elliptic and self-adjoint operators on a smooth manifold without boundary and the equations are driven by a Brownian motion. This theory was developed further by Zabczyk ([19]) in much greater generality (see also the seminal monograph [5]), Peszat ([8, 17]), and other authors ([4], [14]).

The aim of this paper is to extend and complement already existing results for OrnsteinUhlenbeck processes driven by a (cylindrical) Wiener processes that take values in a finiteor infinite-dimensional Hilbert space to Ornstein-Uhlenbeck processes driven by Lévy processes that possibly have jumps. Our results appear to be new also in the case where $H=\mathbb{R}^{d}$ for some $d \in \mathbb{N}$.

We fix a real separable Hilbert space $H$ and a $H$-valued Lévy process $L=\left(L_{t}\right)_{t \geqslant 0}$ on some probability space $(\Omega, \mathcal{F}, \mathrm{P})$ that is expressed in the Lévy-Itô decomposition as $L_{t}=b t+W_{t}+Z_{t}(t \geqslant 0)$, where $b \in H, W=\left(W_{t}\right)_{t \geqslant 0}$ is a (possibly degenerate) Wiener process with the covariance operator $Q$, and $\left(Z_{t}\right)_{t \geqslant 0}$ is the jump part of $L$ (see Theorem 2.5 for more details).

Theorem A. Let $T>0$. Suppose that the eigenvalues of the covariance operator $Q$ corresponding to $W$ are strictly positive. Let $X, \tilde{X}: \Omega \rightarrow \mathcal{D}_{H, T}$ be the Ornstein-Uhlenbeck processes solving

$$
\left\{\begin{aligned}
\mathrm{d} X_{t} & =A X_{t} \mathrm{~d} t+\mathrm{d} L_{t}, \\
\mathrm{~d} \tilde{X}_{t} & =\tilde{A} \tilde{X}_{t} \mathrm{~d} t+\mathrm{d} L_{t}, \\
X_{0} & =0 \\
\tilde{X}_{0} & =0
\end{aligned}\right.
$$

If $H$ is finite-dimensional or

$$
\int_{0}^{t}\left(A X_{s}-\tilde{A} X_{s}\right) \mathrm{d} s \in \operatorname{im} Q^{1 / 2} \quad(t \in[0, T]),
$$

then the laws of $X$ and $\tilde{X}$ are equivalent. 
Note. When the Hilbert space $H$ is finite-dimensional, the main hypothesis of Theorem A is equivalent to invertibility of the covariance matrix $Q$ corresponding to $W$, in which case (1.3) holds vacuously as $Q^{1 / 2}$ is invertible. Otherwise, $Q^{1 / 2}$ is only a densely defined positive operator as $Q$ is trace-class.

In the case where the underlying Lévy process $L$ is a purely jump process, solutions to (1.2) with respect to $L$ exhibit a remarkably rigid behaviour.

Theorem B. Let $T>0$. Suppose that $L$ is a purely jump process, that is, $L=Z$ as written in the Lévy-Itô decomposition. Let $X_{J}, \tilde{X}_{J}: \Omega \rightarrow \mathcal{D}_{H, T}$ be the Ornstein-Uhlenbeck processes solving (1.2). If the law of $X_{J}$ is absolutely continuous with respect to the law of $\tilde{X}_{J}$, then the processes $X_{J}$ and $\tilde{X}_{J}$ are equal to each other almost surely.

\section{Preliminaries}

Let $(\Omega, \mathcal{F}, \mathrm{P})$ be a probability space and let $(S, \mathcal{S})$ be a measurable space. A function $X: \Omega \rightarrow S$ is an $S$-valued random variable when $X^{-1}(E) \in \mathcal{F}$ for every $E \in \mathcal{S}$. The law of $X$ is the pull-back measure $\mathrm{P}_{X}$ on $(S, \mathcal{S})$ given by $\mathrm{P}_{X}(E)=\mathrm{P}\left(X^{-1}(E)\right)(E \in \mathcal{S})$. When $S$ carries the structure of a metric space, by default we will take $\mathcal{S}=$ Bor $S$, the $\sigma$-algebra of Borel subsets of $S$. For two measures $\mu$ and $\nu$ we denote by $\mu \otimes \nu$ the product measure defined on the product $\sigma$-algebra, that is, the smallest $\sigma$-algebra containing all measurable rectangles from the respective measure spaces. For a separable metric space $S$, or more generally, a second-countable Hausdorff space, the product $\sigma$-algebra Bor $S \otimes$ Bor $S$ coincides with Bor $S \times S$ (see, e.g., [3, Lemma 6.4.2]).

2.1. The Skorohod metric. Let $(S, d)$ be a separable metric space and let $T>0$ be given. Let $\mathcal{D}_{S, T}$ denote the space of all $S$-valued càdlàg functions, that is, right-continuous functions $f:[0, T] \rightarrow S$ with the property that for each $t>0$ the left limit at $t, f(t-)$, exists. Denote by $\Lambda_{T}$ the family of all strictly increasing functions $\phi$ from $[0, T]$ onto itself with $\phi(0)=0$ and $\phi(T)=T$. Then the formula

$$
d_{S}(f, g)=\inf _{\phi \in \Lambda_{T}} \max \left\{\sup _{t \in[0, T]}|\phi(t)-t|, \sup _{t \in[0, T]} d(f(\phi(t), g(t))\} \quad\left(f, g \in \mathcal{D}_{S, T}\right)\right.
$$

defines a metric on $\mathcal{D}_{S, T}$, called the Skorohod metric ([10, p. 265 \& Proposition 1.6]). The Borel $\sigma$-algebra of the space of càdlàg functions with the Skorohod metric, Bor $\mathcal{D}_{S, T}$, coincides with the $\sigma$-algebra of cylindrical sets-in other words, it is the smallest $\sigma$-algebra on $\mathcal{D}_{S, T}$ for which the point evaluations $p_{t}(f)=f(t)\left(t \in[0, T], f \in \mathcal{D}_{S, T}\right)$ are measurable ([10, Corollary 2.4]). We shall frequently invoke the following consequence of this fact. 
Proposition 2.1. Let $(\Omega, \mathcal{F})$ be a measurable space. Then a function $X: \Omega \rightarrow \mathcal{D}_{S, T}$ is measurable if and only if, for every $t \in[0, T]$ the composite map $\pi_{t} \circ X$ is measurable.

2.2. Absolute continuity of measures. Let $\mu, \nu$ be measures on a measurable space $(S, \mathcal{S})$. The measure $\mu$ is called absolutely continuous with respect to $\nu$ (in short, $\mu \ll \nu$ ), when $\nu(E)=0(E \in \mathcal{S})$ implies that $\mu(E)=0$. Two measures are equivalent when they are mutually absolutely continuous. Let us record the following corollary to Fubini's theorem concerning absolute continuity of product measures ([7, p. 92]). Suppose that $\mu_{i}, \nu_{i}$ are $\sigma$-finite measures on measurable spaces $\left(S_{i}, \mathcal{S}_{i}\right)$ such that $\mu_{i} \ll \nu_{i}(i=1,2)$. Then $\mu_{1} \otimes \mu_{2} \ll \nu_{1} \otimes \nu_{2}$. We will make use of this fact stated in the following form.

Lemma 2.2. Let $(\Omega, \mathcal{F}, \mathrm{P})$ be a probability space and let $(S, \mathcal{S})$ be a measurable space. Suppose that $X, Y_{1}, Y_{2}: \Omega \rightarrow S$ are random variables such that

(i) $P_{Y_{1}} \ll P_{Y_{2}}$,

(ii) the variables $X, Y_{i}$ are independent $(i=1,2)$.

Then the law $\mathrm{P}_{\left(X, Y_{1}\right)}$ is absolutely continuous with respect to $\mathrm{P}_{\left(X, Y_{2}\right)}$.

Proof. Independence of $X$ and $Y_{i}$ is equivalent to $\mathrm{P}_{\left(X, Y_{i}\right)}=\mathrm{P}_{X} \otimes \mathrm{P}_{Y_{i}}(i=1,2)$ (see, e.g., [15, Théorème IV.1.3]).

2.3. Lévy processes. Let $B$ be a separable Banach space. A stochastically continuous, $B$-valued process $L=\left(L_{t}\right)_{t \geqslant 0}$, is Lévy, when $L_{0}=0$ almost surely, the increments of $L$ are independent and stationary, and almost every sample path $f(t):=L_{t}(\omega)(\omega \in \Omega)$ of $L$ is a $B$-valued càdlàg function.

Let $E \in$ Bor $B \backslash\{0\}, t \geqslant 0$ and $f \in \mathcal{D}_{B}$. We then define

$$
\pi_{t}(E, f)=\operatorname{card}\{s \leqslant t: \Delta f(s):=f(s)-f(s-) \in E\} .
$$

For a Lévy process $L=\left(L_{t}\right)_{t \geqslant 0}$ we may then set

$$
\pi_{t}(E, L)(\omega)=\pi_{t}(E, f) \quad(\omega \in \Omega),
$$

where $f(t)=L_{t}(\omega)$ is a sample path of $L$. Put simply, $\pi_{t}(E, L)(\omega)$ counts the number of jumps in $E$ up to time $t$ that the sample path of $L$ at $\omega$ has in the set $E$. The family $\left\{\pi_{t}(\cdot, L): t \geqslant 0\right\}$ is called the Poisson random measure of $L$. The formula

$$
\mu(E)=\mathrm{E}\left[\pi_{t}(E, L)\right] \quad(E \in \operatorname{Bor} B \backslash\{0\})
$$

defines a Borel measure on $B \backslash\{0\}$, called the intensity measure of $L$. For a bounded below Borel set $E \subset B$, that is a set with $\operatorname{dist}(0, E)>0$, and $f \in \mathcal{D}_{B}$ we set

$$
\hat{\pi}_{t}(E, f)=\pi_{t}(E, f)-t \cdot \mu(E) \quad(t \geqslant 0) .
$$


Whenever $E$ is bounded below, the expression

$$
Z_{E}^{1}(f, t):=\sum_{\substack{0 \leqslant s \leqslant t \\ \Delta f(s) \in E}} \Delta f(s)=\int_{E} u \pi_{t}(\mathrm{~d} u, f)
$$

defines a function in $\mathcal{D}_{B, T}$. If $E$ is also bounded

$$
Z_{E}^{2}(f, t):=Z_{E}^{1}(f, t)-\int_{E} u \mu(\mathrm{d} u)
$$

defines an element in $\mathcal{D}_{B, T}$; we shall be primarily concerned with the $\mathcal{D}_{B, T}$-valued random variables of the form $Z_{E}^{2}(L, t)$.

For every Borel set $E \subset B$ that is bounded below, the map $Z_{E}^{1}: \mathcal{D}_{B, T} \rightarrow \mathcal{D}_{B, T}$ is Borel. We are indebted to Mateusz Kwaśnicki for sharing with us a direct proof of this fact ([12]); this argument replaces our previous, overly roundabout reasoning. Note that Borel measurability of $Z_{E}^{2}$ follows from Borel measurability of $Z_{E}^{1}$ as the former is a translation of the latter function. Let us then record these findings for the future reference.

Lemma 2.3. Fix $T>0$ and $E \subset H$ be a non-empty Borel set that is bounded below. Then, the transformation $Z_{E}^{1}: \mathcal{D}_{B, T} \rightarrow \mathcal{D}_{B, T}$ given by (2.3) is Borel.

When $E$ is also bounded, the same is true for $Z_{E}^{2}: \mathcal{D}_{B, T} \rightarrow \mathcal{D}_{B, T}$ given by (2.4) being a translation of $Z_{E}^{1}$.

Remark 2.4. As observed by Applebaum ([2, Section 4]), for any $t \geqslant 0$ and for every sequence $\left(E_{n}\right)_{n=1}^{\infty}$ of Borel sets in the unit ball $B_{1}$ of $B$ such that $E_{n}^{c}=B_{1} \backslash E_{n}(n \in \mathbb{N})$ is bounded below and the sets $E_{n}$ decrease to $\{0\}$, the random variables

$$
Z_{E_{n}^{c}}^{2}(L, t)=\int_{E_{n}^{c}} \hat{\pi}_{t}(\mathrm{~d} u, L),
$$

converge almost surely as $n \rightarrow \infty$ to a random variable

$$
Z_{B_{1}}^{2}(L, t):=\int_{B_{1}} \hat{\pi}_{t}(\mathrm{~d} u, L)
$$

([1, Section 2.3]; see also [2, p. 80]). Moreover the above limit does not depend on the choice of $\left(E_{n}\right)_{n=1}^{\infty}$.

Under this framework, one recovers the Lévy-Itô decomposition for $B$-valued Lévy processes (see [2, Theorem 4.1], [6, Theorem 2.1], and [18, Theorem 6.3]). 
Theorem 2.5 (Lévy-Itô decomposition). Let B be a separable Banach space and let $\left(L_{t}\right)_{t \geqslant 0}$ be a B-valued Lévy process with the corresponding Poisson random measure

$$
\left\{\pi_{t}(\cdot, L): t \geqslant 0\right\}
$$

that has intensity measure $\mu$. Then there are $b \in B$ and a Wiener process $W_{Q}$ with a (possibly degenerate) covariance operator $Q$ such that

$$
L_{t}=b t+W_{Q}(t)+\int_{B_{1}} \hat{\pi}_{t}(\mathrm{~d} u, L)+\int_{B \backslash B_{1}} \pi_{t}(\mathrm{~d} u, L) \quad(t \geqslant 0) .
$$

We term

$$
Z_{t}=\int_{B_{1}} \hat{\pi}_{t}(\mathrm{~d} u, L)+\int_{B \backslash B_{1}} \pi_{t}(\mathrm{~d} u, L)=Z_{B \backslash B_{1}}^{1}(L, t)+Z_{B_{1}}^{2}(L, t) \quad(t \geqslant 0)
$$

the jump part of $L$. The Wiener process $W_{Q}$ and the jump part $Z$ are independent ([18, Theorem 6.3]).

\section{Proof of Theorem A}

We consider a $H$-valued Lévy process $L=\left(L_{t}\right)_{t \geqslant 0}$ on a probability space $(\Omega, \mathcal{F}, \mathrm{P})$ that is expressed in the Lévy-Itô decomposition as $L_{t}=b t+W_{t}+Z_{t}(t \in[0, T])$, where $b \in H$, $W=\left(W_{t}\right)_{t \geqslant 0}$ is a (possibly degenerate) Wiener process and $\left(Z_{t}\right)_{t \geqslant 0}$ is the jump part of $L$. Let $X, \tilde{X}: \Omega \rightarrow \mathcal{D}_{H, T}$ be the Ornstein-Uhlenbeck processes solving (1.1). Moreover, we consider solutions $X^{J}$ and $\tilde{X}^{J}$ to the auxiliary equations without the Wiener part:

$$
\left\{\begin{array}{l}
\mathrm{d} X_{t}^{J}=A X^{J} \mathrm{~d} t+\mathrm{d}\left(L_{t}-W_{t}\right) \\
\mathrm{d} \tilde{X}_{t}^{J}=\tilde{A} \tilde{X}^{J} \mathrm{~d} t+\mathrm{d}\left(L_{t}-W_{t}\right)
\end{array}\right.
$$

with the initial conditions $X^{J}(0)=\tilde{X}^{J}(0)=0$. Let us take a note that $X^{J}$ and $\tilde{X}^{J}$ (can be modified to) have càdlàg sample paths, which we will employ later.

Proposition 3.1. The processes $X$ and $X^{J}+W$ have equivalent laws.

Proof. Let $\left(\mathcal{F}_{t}^{W}\right)_{t \geqslant 0}$ be the natural filtration of $W$ and let us consider the process

$$
W_{t}^{*}=X_{t}-X_{t}^{J}=\int_{0}^{t} A\left(X_{s}-X_{s}^{J}\right) \mathrm{d} s+W_{t} \quad(t \in[0, T]) .
$$

Then $W^{*}$ is the unique strong solution to $\mathrm{d} W_{t}^{*}=A W_{t}^{*} \mathrm{~d} t+\mathrm{d} W_{t}$ with $W_{0}^{*}=0$ that is adapted to the filtration $\left(\mathcal{F}_{t}^{W}\right)_{t \geqslant 0}$.

Since $Z$ and $W$ are independent processes, so are $Z$ and $W^{*}$. By Girsanov's theorem (see [13, Theorem 1] for a version of Girsanov's theorem for $H$-valued processes; this is where 
we apply the hypothesis that the eigenvalues of the covariance operator are strictly positive as well as (1.3) in the case where $H$ is innfinite-dimensional), there is a probability measure $\tilde{\mathrm{P}}$ for which $W^{*}$ is a Wiener process on $(\Omega, \mathcal{F}, \tilde{\mathrm{P}})$ with the same covariance operator as $W$ and so the laws $\mathrm{P}_{W}$ and $\mathrm{P}_{W^{*}}$ are equivalent. The processes $X^{J}$ and $W$ are independent. Let us observe that the processes $X^{J}$ and $W^{*}$ are independent too. Indeed, $W^{*}$ being adapted to $\left(\mathcal{F}_{t}^{W}\right)_{t \geqslant 0}$ is $\mathcal{F}^{W}$-measurable, and thus independent from $\left(X_{t}^{J}\right)_{t \geqslant 0}$ (see also [9, Theorem II.6.3]).

We are now in a position to apply Lemma 2.2 to conclude that the laws $\mathrm{P}_{\left(X^{J}, W\right)}$ and $\mathrm{P}_{\left(X^{J}, W^{*}\right)}$ are equivalent. Consequently, the laws $\mathrm{P}_{X^{J}+W}$ and $\mathrm{P}_{X^{J}+W^{*}}=\mathrm{P}_{X}$ are equivalent as well, which completes the proof.

Thus, in order to establish Theorem A, it is enough to prove the following proposition.

Proposition 3.2. The processes $X^{J}+W$ and $\tilde{X}^{J}+W$ have equivalent laws.

Proof. We will demonstrate that the law of $X^{J}+W$ is absolutely continuous with respect to the law of $\tilde{X}^{J}+W$ as the other direction would be completely analogous.

For given $R>0$ consider the set

$$
\Omega_{R}:=\left\{\omega \in \Omega: \sup _{t \in[0, T]}\left\|A X_{t}(\omega)-\tilde{A} \tilde{X}_{t}(\omega)\right\| \leqslant R\right\}
$$

and note that $\Omega_{R} \in \mathcal{F}$ (cf. Proposition 2.1). We may then consider the 'truncated' process

$$
W_{t}^{R}=W_{t}+\int_{0}^{t}\left(A X_{s}-\tilde{A} \tilde{X}_{s}\right) \cdot \mathbb{1}_{\Omega_{R}} \mathrm{~d} s \quad(t \in[0, T]) .
$$

By hypothesis (1.3), we may apply Girsanov's theorem, so there is a probability measure $\mathrm{P}^{R}$ equivalent to $\mathrm{P}$, for which $W^{R}$ is a Wiener process on $\left(\Omega, \mathcal{F}, \mathrm{P}^{R}\right)$ with the same covariance operator as $W$; in particular the laws $\mathrm{P}_{W}$ and $\mathrm{P}_{W^{R}}$ are equivalent. Arguing as in the proof of Proposition 3.2, we infer that the processes $X$ and $W^{R}$ are independent. By Lemma 2.2 applied to $\left(\tilde{X}^{J}, W\right)$ and $\left(\tilde{X}^{J}, W^{R}\right)$, we deduce that the processes $\tilde{X}^{J}+W$ and $\tilde{X}^{J}+W^{R}$ have equivalent laws.

Since

$$
X_{t}^{J}-\tilde{X}_{t}^{J}=\int_{0}^{t}\left(A X_{s}-\tilde{A} \tilde{X}_{s}\right) \mathrm{d} s
$$

we see that $\tilde{X}_{t}^{J}+W_{t}^{R}$ and $X_{t}^{J}+W_{t}$ agree on the set $\Omega_{R}$. It follows that for every set $E \in \operatorname{Bor} \mathcal{D}_{H, T}$ the condition $\mathrm{P}\left(\tilde{X}_{t}^{J}+W_{t}^{R} \in E\right)=0$ implies that for all numbers $R>0$ we have $\mathrm{P}\left(\Omega_{R} \cap\left(\tilde{X}_{t}^{J}+W\right)\right)=0$. The processes $X^{J}, \tilde{X}^{J}$ have càdlàg sample paths, which 
implies that they are bounded on bounded intervals. In particular, for any $\omega \in \Omega$ the function

$$
t \mapsto A X_{t}(\omega)-\tilde{A} \tilde{X}_{t}(\omega) \quad(t \in[0, T]),
$$

is bounded, which means that $\Omega=\bigcup_{R>0} \Omega_{R}$. Thus $\mathrm{P}_{X^{J}+W} \ll \mathrm{P}_{\tilde{X}^{J}+W}$.

\section{Proof of Theorem B}

This time we consider a purely jump Lévy process $L$, that is $L=Z$ using the notation of Theorem 2.5. Let $X^{J}, \tilde{X}^{J}: \Omega \rightarrow \mathcal{D}_{H, T}$ be solutions to (1.1), which now take the form

$$
\left\{\begin{array}{l}
\mathrm{d} X_{t}^{J}=A X_{t}^{J} \mathrm{~d} t+\mathrm{d} Z_{t}, \\
\mathrm{~d} \tilde{X}_{t}^{J}=\tilde{A} \tilde{X}^{J}{ }_{t} \mathrm{~d} t+\mathrm{d} Z_{t}
\end{array} \quad(t \in[0, T])\right.
$$

with the initial conditions $X_{0}^{J}=\tilde{X}_{0}^{J}=0$.

Lemma 4.1. For any $t \in[0, T]$ and $E \in$ Bor $H \backslash\{0\}$ we have

$$
\mathrm{P}\left(\pi_{t}\left(E, X_{J}\right)=\pi_{t}(E, Z)\right)=1 .
$$

Proof. Since

$$
X_{t}^{J}=A \int_{0}^{t} X_{s}^{J} \mathrm{~d} s+Z_{t}
$$

we have

$$
\begin{aligned}
\left\{s \leqslant t: X_{s}^{J}-X_{s-}^{J} \in E\right\} & =\left\{s \leqslant t: A \int_{0}^{s} X_{u}^{J} \mathrm{~d} u+Z_{s}-A \int_{0}^{s-} X_{u}^{J} \mathrm{~d} u-Z_{s-} \in E\right\} \\
& =\left\{s \leqslant t: Z_{s}-Z_{s-} \in E\right\}
\end{aligned}
$$

almost surely.

We may then derive the following conclusion.

Corollary 4.2. For every non-negative integer $k, t \in[0, T]$, and $E \in \operatorname{Bor} H \backslash\{0\}$ we have

$$
\mathrm{P}_{X^{J}}\left(\left\{f \in \mathcal{D}_{H, T}: \pi_{t}(E, f)=k\right\}\right)=\mathrm{P}\left(\pi_{t}\left(E, X^{J}\right)=k\right)=\mathrm{P}\left(\pi_{t}(E, Z)=k\right) .
$$

In particular, for a sequence $\left(E_{n}\right)_{n=1}^{\infty}$ of Borel sets in the unit ball $H$, as in the statement of Remark 2.4, the random variables $Z_{E_{n}^{c}}^{2}\left(X^{J}, t\right)$ converge almost surely to $Z_{H_{1}}^{2}(Z, t)$ as $n \rightarrow \infty(t \in[0, T])$.

For $t \in[0, T]$ and $f \in \mathcal{D}_{H, T}$ we set

$$
S(f, t)=\int_{0}^{t} f(s) \mathrm{d} s
$$


Consequently, the assignment $f \mapsto S(f, \cdot)$ defines a function $\mathcal{D}_{H, T} \rightarrow \mathcal{D}_{H, T}$ as it takes continuous values.

Lemma 4.3. For a bounded linear operator $V: H \rightarrow H$, the assignment $\mathcal{D}_{H, T} \rightarrow \mathcal{D}_{H, T}$ given by $f \mapsto V S(f, \cdot)\left(f \in \mathcal{D}_{H, T}\right)$ is Borel.

Proof. Observe that if a sequence $\left(f_{n}\right)_{n=1}^{\infty}$ in $\mathcal{D}_{H, T}$ converges to some $f \in \mathcal{D}_{H, T}$, then for almost all $s \in[0, T]$ (with respect to the Lebesgue measure) we have $f_{n}(s) \rightarrow f(s)$ as $n \rightarrow \infty$. Since the sequence $\left(f_{n}\right)_{n=1}^{\infty}$ is bounded with respect to the supremum norm, by the dominated convergence theorem (for Bochner-integrable functions) we conclude that for all $t \in[0, T]$

$$
\int_{0}^{t} f_{n}(s) \mathrm{d} s \rightarrow \int_{0}^{t} f(s) \mathrm{d} s
$$

as $n \rightarrow \infty$. Consequently, the map $\Phi(f)=V S(\cdot, f)\left(f \in \mathcal{D}_{H, T}\right)$ is Borel-measurable because it is continuous as a map from $\mathcal{D}_{H, T}$ with the Skorohod topology to $\mathcal{D}_{H, T}$ (actually even to $C([0, T], H))$ endowed with the topology of pointwise convergence. Indeed, by Proposition 2.1, $\Phi$ is Borel because for each $t \in[0, T]$, the map $\Phi(\cdot)(t): \mathcal{D}_{H, T} \rightarrow H$ is continuous, hence Borel.

Definition 4.4. For a bounded linear operator $V: H \rightarrow H$ we define $\Xi_{V}=\left\{f \in \mathcal{D}_{H, T}: \lim _{n \rightarrow \infty}\left\|f(t)-V S(f, t)-Z_{H \backslash H_{1}}^{1}(f, t)-Z_{E_{n}^{c}}^{2}(f, t)\right\|=0\right.$ for all $\left.t \in[0, T]\right\}$.

By Lemma 4.3, the assignment $f \mapsto V S(f, \cdot)\left(f \in \mathcal{D}_{H, T}\right)$ is Borel measurable. Similarly, by Lemma 2.3, the assignments $f \mapsto Z_{E}^{1}(f, \cdot), Z_{E}^{2}(f, \cdot)\left(f \in \mathcal{D}_{H, T}\right)$ are Borel measurable for every bounded below set $E \subset H$ (in the latter case, $E$ is assumed to be additionally bounded). Let us invoke Proposition 2.1 to see that in order to establish measurability of $\Xi_{V}$ it is enough to show measurability of $p_{t}\left[\Xi_{V}\right]$ for each $t \in[0, T]$. We have thus proved the following proposition.

Proposition 4.5. For a bounded linear operator $V: H \rightarrow H$, the set $\Xi_{V}$ is Borel with respect to the Skorohod topology on $\mathcal{D}_{H, T}$.

Lemma 4.6. $\mathrm{P}_{X^{J}}\left(\Xi_{A}\right)=1=\mathrm{P}_{\tilde{X}^{J}}\left(\Xi_{\tilde{A}}\right)$.

Proof. By Corollary 4.2, $Z_{H \backslash H_{1}}^{1}\left(X^{J}, t\right)+Z_{E_{n}^{c}}^{2}\left(X^{J}, t\right)$ converges almost surely as $n \rightarrow \infty$ to $Z(L, t)(t \in[0, T])$. Thus, $\mathrm{P}_{X^{J}}\left(\Xi_{A}\right)$ is equal to

$$
\mathrm{P}\left(X_{t}^{J}-A S\left(X^{J}, t\right)=Z\left(X^{J}, t\right)(t \in[0, T])\right)=\mathrm{P}\left(X_{t}^{J}-A \int_{0}^{t} X_{s}^{J} \mathrm{~d} s=Z_{t}(t \in[0, T])\right)=1 .
$$

The same proof applies for $\Xi_{\tilde{A}}$. 
We are now ready to prove Theorem B.

Proof of Theorem B. Assume that $\mathrm{P}_{X^{J}} \ll \mathrm{P}_{\tilde{X}^{J}}$. By Lemma 4.6, $\mathrm{P}_{X^{J}}\left(\Xi_{A}\right)=1=\mathrm{P}_{\tilde{X}^{J}}\left(\Xi_{\tilde{A}}\right)$. Consequently, by absolute continuity we must have $\mathrm{P}_{X^{J}}\left(\Xi_{\tilde{A}}\right)=1$, which means that

$$
X_{t}^{J}=\tilde{A} \int_{0}^{t} X_{s}^{J} \mathrm{~d} s+Z_{t} \quad(t \in[0, T])
$$

almost surely. We have thus proved that $X^{J}$ solves the stochastic differential equation $\mathrm{d} Y_{t}=A Y_{t} \mathrm{~d} t+\mathrm{d} Z_{t}$, so by the uniqueness of solutions, $X^{J}=\tilde{X}^{J}$ almost surely.

4.1. Closing remarks. In the case of stochastic processes in infinite dimensions it is customary to work with generators of infinitesimal semigroups rather than merely bounded linear operators. However, the proof methods employed in this paper required the operators $A$ and $\tilde{A}$ appearing in (1.1) to be bounded ( $c f$. the proofs of Proposition 3.2 and Lemma 4.3). It is thus natural to ask whether Theorems A and B have their counterparts in the setting of generators of infinitesimal semigroups too. From this point of view, it is also desirable to investigate classes of those Feller processes for which analogous results can be established.

Acknowledgements. We wish to express our gratitude to Mateusz Kwaśnicki (Wrocław) for sharing with us a direct proof of Lemma 2.3. Furthermore, we are greatly indebted to the anonymous referee for spotting the need for the technical assumption (1.3) that is indeed required when the underlying Hilbert space is infinite-dimensional.

\section{REFERENCES}

[1] D. Applebaum, Lévy processes and stochastic calculus, Cambridge University Press, 2004.

[2] D. Applebaum, Lévy processes and stochastic integrals in Banach Spaces, Prob. Math. Stat., 27 (2007), 75-88.

[3] V. I. Bogachev, Measure Theory, Volume II, Springer, 2006.

[4] Z. Brzeźniak and J. van Neerven, Equivalence of Banach space-valued Ornstein-Uhlenbeck processes, Stochastics Stochastics Rep. 69 (2000), 77-94.

[5] G. Da Prato and J. Zabczyk, Stochastic equations in infinite dimensions, Cambridge University Press, 1992.

[6] E. Dettweiler, Banach space valued processes with independent increments and stochastic integration, in: Probability in Banach Spaces, IV Proceedings Oberwolfach 1982, A. Beck and K. Jacobs (Eds.), Lecture Notes in Math. No 990, Springer, Berlin 1983, 54-84.

[7] G. B. Folland, Real analysis. Modern techniques and their applications. Second edition. Pure and Applied Mathematics (New York). A Wiley-Interscience Publication. John Wiley \& Sons, Inc., New York, 1999.

[8] B. Gołdys and S. Peszat, Law equivalence of stochastic linear systems, Stat. Probab. Lett. 43, No.3 (1999), 265-274.

[9] N. Ikeda and S. Watanabe, Stochastic Differential Equations and Diffusion Processes, North-Holland, Amsterdam, 1981. 
[10] A. Jakubowski, On the Skorokhod topology, Ann. Inst. Henri Poincaré, Probabilité s et Statistiques, 22 (1986), 263-285.

[11] S. M. Kozlov, Equivalence of measures in linear Itô partial differential equations, Moscow Univ. Mathem. Bull., 32 (1970), 36-40.

[12] M. Kwaśnicki, Transformations of càdlàg functions, https://mathoverflow.net/a/291729/15129, (2018).

[13] W. Loges, Girsanov's theorem in Hilbert space and an application to the statistics of Hilbert spacevalued stochastic differential equations, Stoch. Process. Appl., 17, Issue 2 (1984), 243-263.

[14] B. Maslowski and J. van Neerven, Equivalence of laws and null controllability for SPDEs driven by a fractional Brownian motion, Nonlinear Differential Equations and Applications, 20 (2013), 1473-1498.

[15] M. Métivier, Notions fondamentales de la théorie des probabilités, Dunod, Paris, 1971.

[16] S. Peszat, Equivalence of distributions of some Ornstein-Uhlenbeck processes taking values in Hilbert space, Prob. Math. Stat., 13 (1992), 7-17.

[17] S. Peszat, Law equivalence of solutions of some linear stochastic equations in Hilbert spaces, Studia Math., 101 (1992), 269-284.

[18] M. Riedle and O. van Gaans, Stochastic integration for Lévy processes with values in Banach spaces, Stoch. Anal. Appl. 119 (2009), 1952-1974.

[19] J. Zabczyk, Law equivalence of Ornstein-Uhlenbeck processes, Gaussian Random Fields (Nagoya, 1990), 420-432, Ser. Probab. Statist., 1, World Sci. Publ., River Edge, NJ, 1991.

Korczaka 6, 43-100 Tychy, Poland

E-mail address: grzegorzjanbartosz@wp.pl

Institute of Mathematics, Czech Academy of Sciences, Žitná 25, 11567 Prague 1, Czech REPUBLIC

E-mail address: tomasz.marcin.kania@gmail.com 